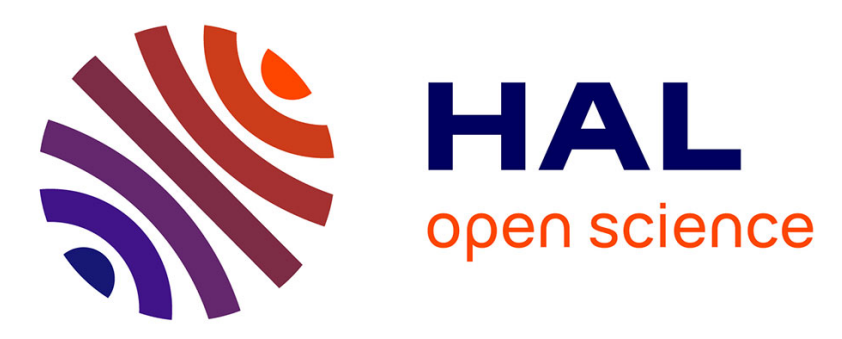

\title{
Détente mécanique et dynamique de versant dans l'Eocène de l'est du bassin de Paris, France
}

Nicolas Bollot, Alain Devos, Guillaume Pierre, Laurent Chalumeau, Olivier Lejeune

\section{- To cite this version:}

Nicolas Bollot, Alain Devos, Guillaume Pierre, Laurent Chalumeau, Olivier Lejeune. Détente mécanique et dynamique de versant dans l'Eocène de l'est du bassin de Paris, France. Géomorphologie: relief, processus, environnement, 2014. hal-03277151

\section{HAL Id: hal-03277151 \\ https://hal.science/hal-03277151}

Submitted on 2 Jul 2021

HAL is a multi-disciplinary open access archive for the deposit and dissemination of scientific research documents, whether they are published or not. The documents may come from teaching and research institutions in France or abroad, or from public or private research centers.
L'archive ouverte pluridisciplinaire $\mathbf{H A L}$, est destinée au dépôt et à la diffusion de documents scientifiques de niveau recherche, publiés ou non, émanant des établissements d'enseignement et de recherche français ou étrangers, des laboratoires publics ou privés. 


\title{
Détente mécanique et dynamique de versant dans l'Eocène de l'est du bassin de Paris, France Mechanical release and slope dynamics in Eocene strata (eastern Paris basin, France)
}

\author{
Nicolas Bollot*, Alain Devos*, Guillaume Pierre*, Laurent Chalumeau*, Olivier Lejeune*
}

\begin{abstract}
Résumé
La déformation des calcaires lutétiens des plateaux du Tardenois et du Soissonnais est analysée à partir de sondages et d'observations en carrières souterraines. En bordure de plateau, un phénomène de détente, marqué par une fracturation et des pendages anormaux, entraîne un fléchissement des bancs calcaires vers les vallées. Sur les versants, drapés par des blocs calcaires jusqu'en fond de vallée, l'abaissement de la strate résistante de surface s'est fait par érosion des sables cuisiens sous-jacents, probablement dans un contexte périglaciaire. Cette cambrure de versant, associée à l'incision des vallées, est un processus ancien, conduisant à des versants stables.
\end{abstract}

Mots clés : dynamique des versants, détente mécanique, cambrure, Éocène, bassin de Paris.

\begin{abstract}
Deformation of the Lutetian limestone of the Tardenois and Soissonnais plateaux has been surveyed using boreholes and observations in underground quarries. At the edge of the plateaux mechanical release, underlined by fracturing and uneven dips, results in a valleywards bending of calcareous beds. The valley sides are swathed with Lutetian blocks from the top to the foot of the slope. The lowering of the competent surface stratum is due to the downslope creep of the underlying Cuisian sands, probably under periglacial conditions. This valley-side cambering, associated with valley downcutting, is an old process, leading to stable slopes.
\end{abstract}

Key words: slope processes, mechanical release, cambering, Eocene, Paris basin.

\section{Abridged English version}

The wide valleys of the Eocene plateaux of Soissonnais and Tardenois (fig. 1) are cut through Lutetian limestones into underlying Cuisian sands and Sparnacian clay. Valley sides (part of the Champagne vineyard) often have average slopes less than $10^{\circ}$ and have smoothly undulating profiles. Observations in underground quarries at the edge of the plateau show that the "Calcaires grossiers" of the middle Lutetian, affected by a dense fissure network parallel to the valleyside, break up into blocks that swathe the slope towards the valley floor. This paper deals with the relations between the deformations of the Lutetian strata and the morphostructure on the one hand, and with the slope processes influenced by superficial structure on the other hand. Investigations on the deformations of the Lutetian, on the interfluves, are based on borehole data and on direct observations in underground quarries. On the valley side, superficial structure analysis derives from the description of cuts, and from the interpretation of topographic data and of aerial views.
The Eocene formations of the back slope of the Ile-deFrance cuesta gently dip westwards, but neither the general dip, nor secondary tectonic deformations explain the detail geometry of the Lutetian strata (fig. 2). Near the edge of the plateau, the bending of the bottom of the Lutetian comprises between $9.3 \%$ and $1 \%$, with an average of $3.3 \%$ (fig. 5). In underground galleries, the fracture index (number of fissures per metre), taking into account the tension cracks running parallel to the valley side, is 0.15 fissure. $m^{-1}$, i.e. an average distance of $8.46 \mathrm{~m}$ between each crack (Devos et al., 2012). Nearer the valley side slope, the fracture index rises to $0.3 \mathrm{~m}^{-1}$, and the distance between cracks lowered to $5 \mathrm{~m}$ (fig. 6). This means that open cracks are clearly associated with valley cutting and stress relief. The width of the cracks varies between $50 \mathrm{~cm}$ and $2 \mathrm{~m}$, and the open space is generally infilled with overlying "Marnes et caillasses" of the upper Lutetian. Along with the increasing amount of cracks, anomalous dips and tilting of the calcareous blocks are underlined by the shift of key levels (chert, shelly limestone and clayey bed) observed in the galleries perpendicular to the

\footnotetext{
* EA 3795 GEGENA - Département de Géographie - Université de Reims Champagne-Ardenne - 57, rue Pierre Taittinger - 51096 Reims cedex France (nicolas.bollot@univ-reims.fr ; alain.devos@univ-reims.fr ; guillaume.pierre@univ-reims.fr ; laurent.chalumeau@univ-reims.fr ; olivier.lejeune@univ-reims.fr).
} 
valley side (fig. 7). In the three studied quarries, anomalous dips (up to 15\%) are more especially remarkable as they are not in accordance with the regional dip.

The straight profile of the scarp face of the cuesta and of the southern valley side of the river Vesle is draped with separated limestone blocks whose dip is parallel to the slope (between $8^{\circ}$ and $10^{\circ}$ ). On the valley sides of the river Ardre, whitish surfaces identified on vertical airviews correspond to back-tilted blocks that impart a benched profile to the slopes. In this case, slope angle is lesser (between $5^{\circ}$ to $6^{\circ}$ ) (fig.8). Following the diminishing gradient of the foot slope, large blocks undergoing shallow-seated translational movements have greater upslope dip $\left(30^{\circ}\right)$.

As described, cambering cannot be explained by the sliding of calcareous blocks on underlying incompetent strata (Sosson et al., 2011), nor solely by a lateral spread (Pasuto and Soldati, 1996), because these processes do not account for the fact that calcareous blocks may swathe the whole slope. A better explanation is the progressive vertical lowering of the caprock as the valley deepens. This process implies an erosion of the underlying incompetent formations. In the case of impervious clayey material, sub-surface erosion may be attributed to leaching by infiltrated water (Hawkins and Privett, 1979), or to the outflow (Hollingworth et al., 1944), or also the deformation (Hutchinson and Coope, 2002) of plastic rocks conditioned by stress release (Vaughan, 1976; Hobbs and Jenkins, 2008). It has also been suggested that the sub-surface erosion is enhanced by periglacial processes (Parks, 1991; Hutchinson, 1991). As Cuisian sands are intrinsically pervious and not susceptible to compaction-unloading processes, their erosion might only be achieved by freeze-thaw action. These periglacial conditions imply a pre-Holocene age for cambering. Furthermore, the perched position of truncated cambering indicates an even older age, at least pre-Saalian (fig. 9), consistent with estimations by Horswill and Horton (1976), and also with the 350 ka radiometric age of speleothems deposited after mechanical release in Bathonian limestone of the Cotswold hills (Self, 1995). This probable Elsterian minimum age means that cambering leads to stable slopes, contrary to landsliding which threaten the vineyard (Marre et al., 1997).

In France, the first description of outflow of clay layers, sinking and bending in Jurassic strata of the eastern Paris basin is provided by A. Buvignier (1852). Later, P. Maubeuge (1949) mentions valley-side cambering as an ignored problem of applied geology, and J. Tricart (1952) describes the process as contemporary landsliding. Indeed, the excessive thickness of the Jurassic limestone of the eastern Paris basin is not disposed to cambering. In Eocene strata, on the contrary, the thickness-ratio between the "Calcaires grossiers" of the middle Lutetian (15 m near Fismes) and the underlying Cuisian sands $(20$ to $30 \mathrm{~m})$ is favorable, and here cambering prevails.

\section{Introduction}

Dans l'est du bassin de Paris, les vallées qui entaillent les plateaux calcaires de l'Éocène du Soissonnais et du Tardenois (fig. 1) ne présentent pas le même profil transversal que celles des plateaux calcaires de l'auréole jurassique. Quelle que soit la surface des bassins versants retenus, la largeur des vallées est plus importante, et les versants, longs et en pente douce, sont marqués, localement, par des ressauts de faible ampleur (fig. 2). L'Aisne et la Vesle, allochtones, sont encaissées de $130 \mathrm{~m}$ et $160 \mathrm{~m}$, respectivement, et l'Ardre, autochtone, de $150 \mathrm{~m}$. L'étude des discontinuités observées en carrières souterraines (fig. 1) montre que le calcaire lutétien, sur les rebords de plateau, est affecté par un dense réseau de fissures parallèles aux versants, qui calibre des blocs désolidarisés que l'on retrouve jusqu'en fond de vallée. Dans une région où les mouvements de masse, sinon les glissements rotationnels, sont considérés comme les processus morphogéniques dominants (Marre et al., 1997 ; Van Den Eeckaut et al., 2010), la cambrure de versant (valleyside cambering), depuis longtemps décrite par les auteurs anglais (Hollingworth et al., 1944), au point de faire l'objet de parties distinctes dans les manuels (Selby, 1993 ; Ballantyne et Harris, 1994), reste inexplorée. Le terme désigne le fléchissement et l'étirement d'une strate cohérente sur un versant par érosion des couches tendres, généralement argileuses, sous-jacentes. La cambrure s'accompagne d'une fracturation ouverte débitant des trains de blocs qui drapent les versants (fig. 3 ).

En France, il revient sans doute à A. Buvignier (1852) d'avoir décrit pour la première fois un mode d'évolution des versants, à l'Actuel, par « reflux » d'assises argileuses, affaissement et courbure, dans le Jurassique de la Côte de Bar ; mais il faut attendre le milieu du $\mathrm{XX}^{\mathrm{e}}$ siècle pour que la cambrure de versant, " problème méconnu de géologie appliquée » et « champ de recherche vierge » en France, soit évoquée (Maubeuge, 1949). Cet appel n'a cependant eu qu'un faible écho, puisque les seules mentions faites de processus apparentés sont dues à J. Tricart (1952) et à A. Guilcher et J. Tricart (1954), toujours dans le Jurassique de l'est du bassin de Paris. Encore ces processus sont-ils considérés comme actuels (ou post-glaciaires ; Tricart, 1977), et classés parmi les glissements de terrain. L'article s'interroge donc sur les relations entre les déformations de l'étage lutétien et la morphogénèse à l'échelle des versants.

\section{Contexte morphostructural et méthodologie}

Les formations éocènes des plateaux du Tardenois et du Soissonnais, sur le revers de la cuesta d'Ile-de-France, présentent un dispositif structural monoclinal à faible pendage ouest. Le Tardenois constitue l'extrémité nord de la surface infra-stampienne exhumée (surface de Brie ; Dewolf et Pomerol, 1997), dont les argiles à meulière, issues d'une météorisation chimique polyphasée, pré-stampienne puis quaternaire, reposent sur le Bartonien. Le Soissonnais, débarrassé du Bartonien, est un plateau structural calé sur le sommet du Lutétien. Les entailles quaternaires, dans les deux pays, atteignent les argiles de l'Yprésien inférieur (Sparnacien), et les versants des vallées, essentiellement développés dans les sables de l'Yprésien supérieur (Cuisien), sont dominés par les Calcaires grossiers du Lutétien moyen. Seules la Vesle et l'Aisne, aux percées cataclinales, coulent sur la 


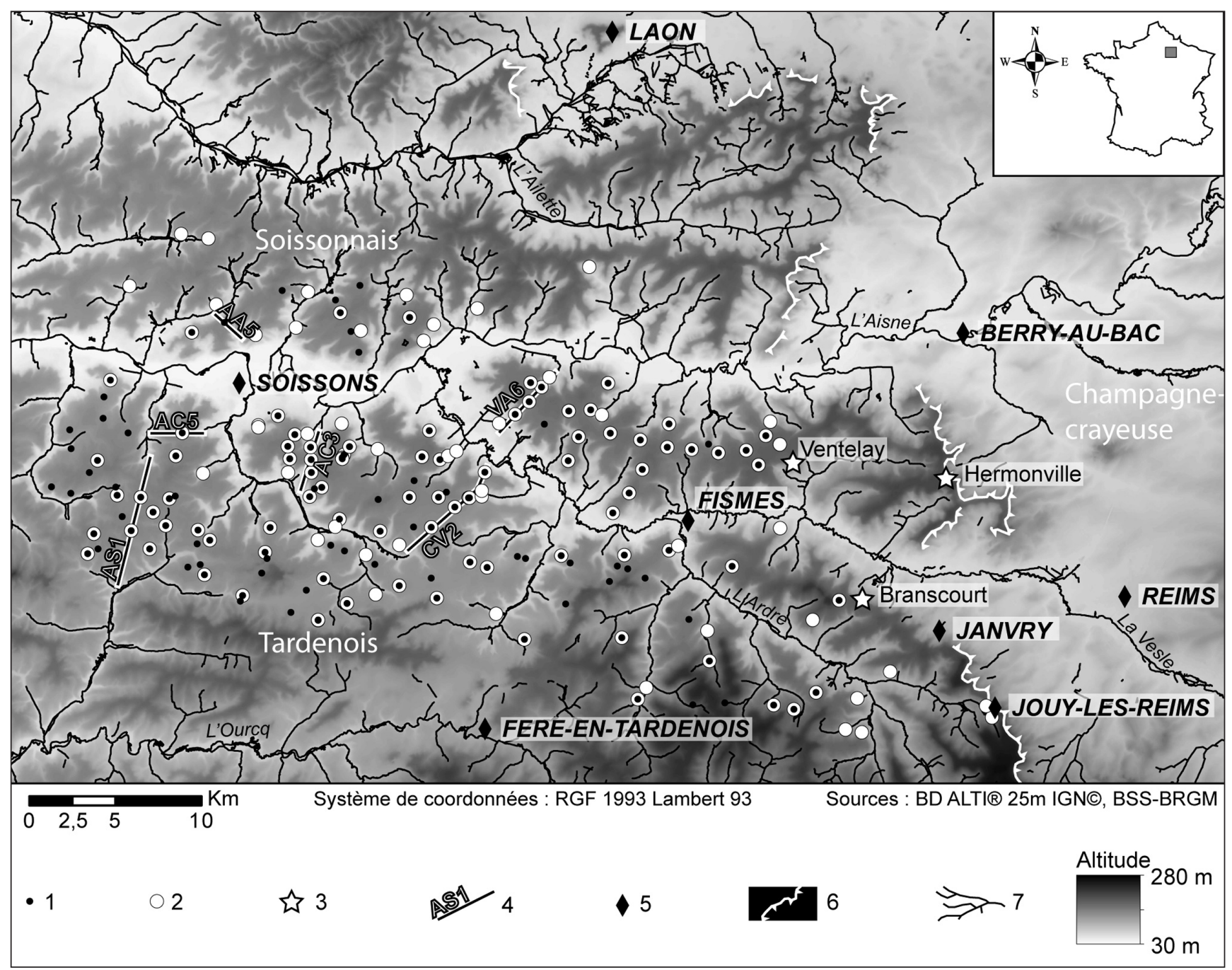

Fig. 1 - Carte de localisation. 1 : sondage atteignant le mur du Lutétien ; 2 : sondage atteignant le mur du Cuisien ; 3 : carrière souterraine étudiée ; 4 : coupe ; 5 : localité ; 6 : cuesta d'lle-de-France ; 7 : cours d'eau.

Fig. 1 - Location map. 1: borehole reaching the bottom of the Lutetian; 2: borehole reaching the bottom of the Cuisian; 3: studied underground quarries; 4: cross section; 5: locality; 6: Ile-de-France cuesta; 7: streams.

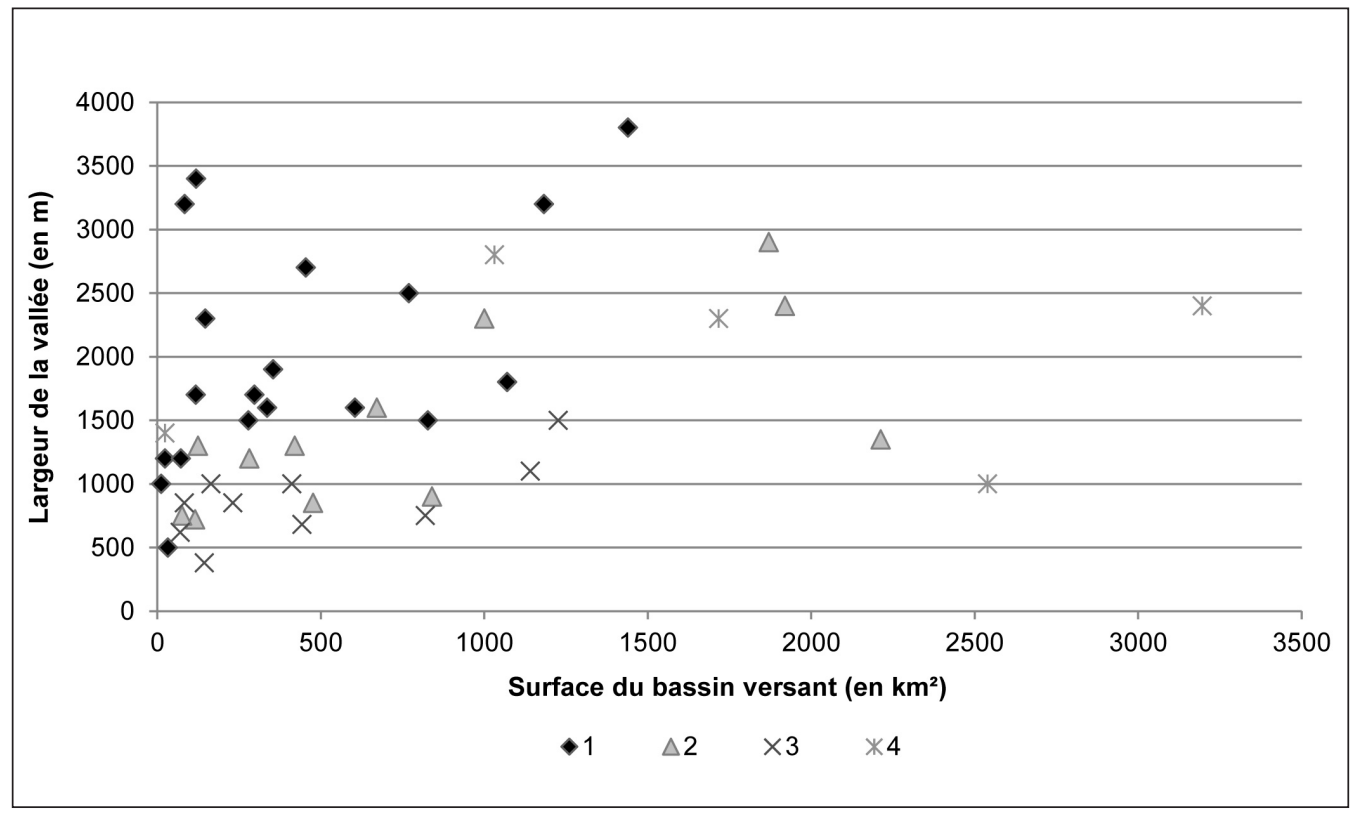

Fig. 2 - Corrélation entre la largeur des vallées et la surface des bassins versants de l'est du bassin de Paris, par ensembles stratigraphiques. 1 : dans l'Eocène ; 2 : dans le Tithonien ; 3 : dans le Bajo-Bathonien ; 4 : dans l'Oxfordien.

Fig. 2 - Correlation between the width of the valleys and the surface of the watersheds of eastern Paris basin, according to stratigraphy. 1: Eocene strata; 2: Tithonian strata; 3: Bajo-Bathonian strata; 4: Oxfordian strata. 


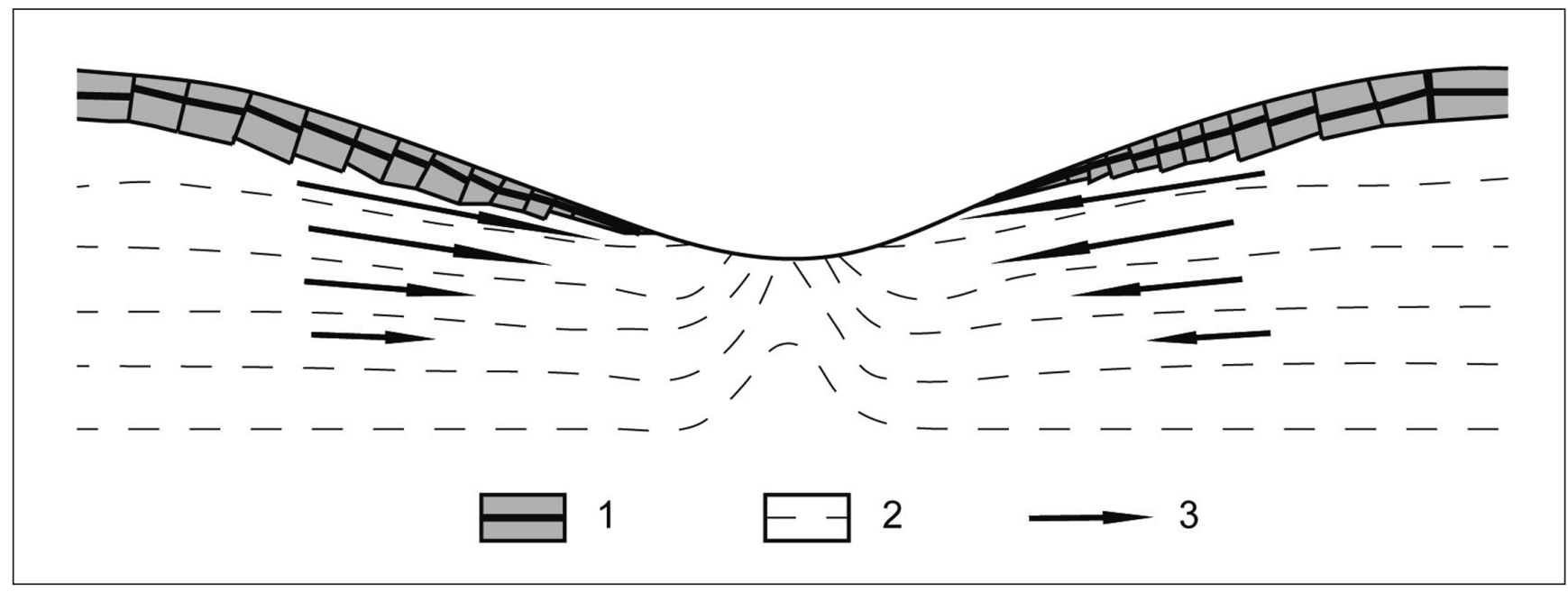

Fig. 3 - Cambrure de versant (d'après Parks, 1991). 1 : roche cohérente avec banc repère (calcaires) ; 2 : roche meuble (argiles) ; $3:$ sens du fluage.

Fig. 3 - Cambering (after Parks, 1991). 1: competent cap-rock with key bed (limestones); 2: soft rock (clays); 3: creep movement.

craie crétacée de la Champagne crayeuse. Dans le Tardenois, le recul de la couverture de Marnes et caillasses du Lutétien supérieur (et des formations bartoniennes sus-jacentes) ménage, en bordure de plateau, un replat structural défini par le toit des Calcaires grossiers du Lutétien moyen.

La déformation des calcaires du Lutétien est analysée, d'un rebord de plateau à l'autre, à partir de forages et d'observations directes en carrières souterraines. Sur les versants, la structure superficielle est déduite de la description de coupes, complétée par l'analyse des formes et par la photo-interprétation. À l'échelle des interfluves, sur des distances kilométriques, la géométrie des couches a été reconstituée à partir 1) des données de sondages sur le plateau (fig. 1) disponibles dans la BSS (Banque du Sous-Sol), et 2) des altitudes d'affleurement indiquées sur les cartes géologiques au 1/50 000 numérisées du BRGM. L'ensemble est regroupé dans une banque de données sous SIG. Pour les 136 sondages utilisés, correspondant à ceux du secteur dont les données sont validées et dont les informations associées sont consultables, la profondeur du mur des calcaires du Lutétien au sein des plateaux est indiquée. À ces données sont ajoutées celles issues des limites du mur des calcaires du Lutétien extraites des cartes géologiques (Laurentiaux et al., 1972 ; Laurain et al., 1976 ; Salin et al., 1977 ; Dorigny et al., 1983). En superposant ces informations à un MNT, il est possible de calculer l'altitude en surface de chaque forage ainsi que l'altitude du mur des calcaires du Lutétien. Cette dernière est alors interpolée par la méthode bilinéaire dite « du plus proche voisin ». Cette méthodologie est la

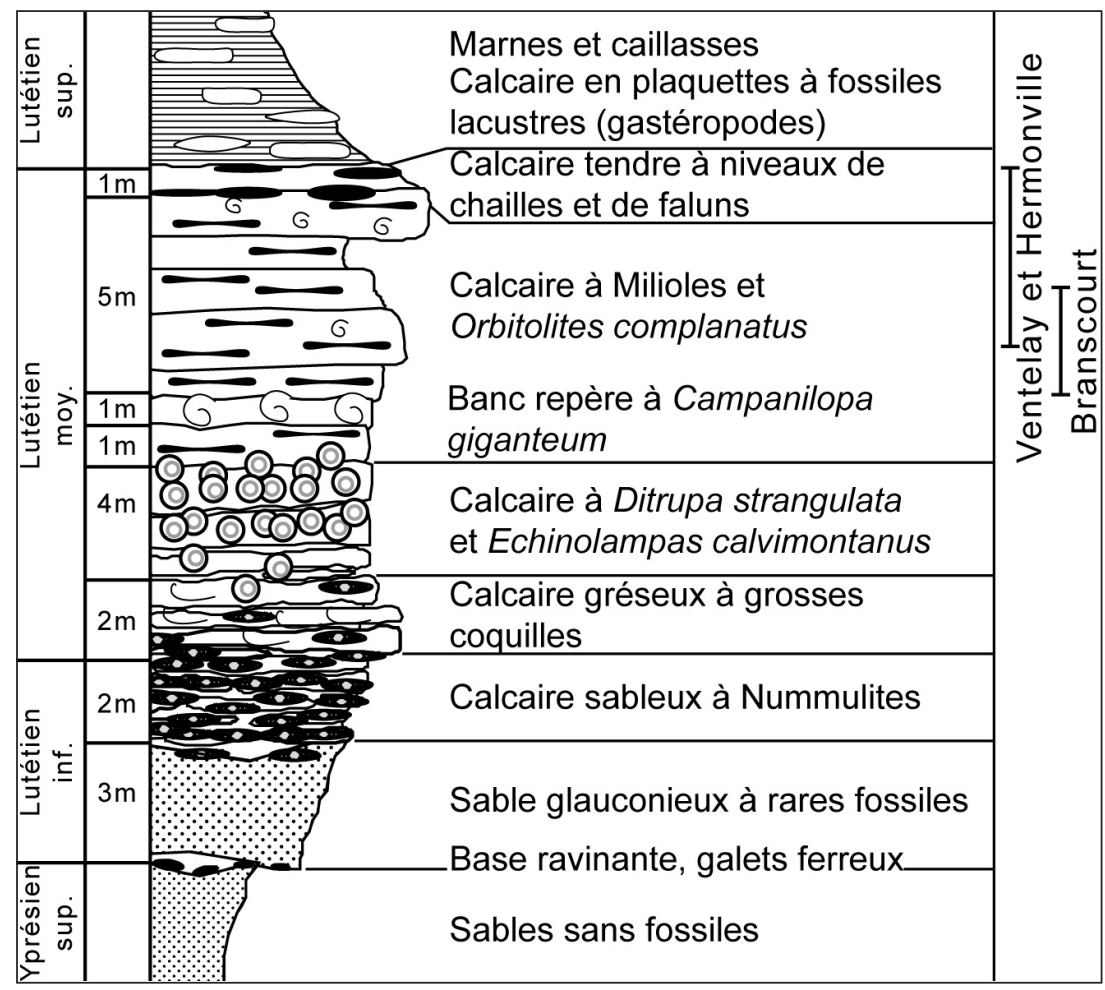

Fig. 4 - Lithostratigraphie du Lutétien à Hermonville (d'après Fronteau et al., 2002, modifié).

Fig. 4 - Lithostratigraphy of the Lutetian of Hermonville (after Fronteau et al., 2002, modified). 
des vallées (Blondeau, 1965 ; Moiriat et al., 2005 ; Devos et al., 2012). Sur plusieurs centaines de cavités dans le secteur d'étude, 32 ont fait l'objet d'une cartographie, de mesures d'azimut magnétique des fissures recoupées par les cavités, ainsi que de mesures de largeur des fissures et de distance entre fissures. Seules les discontinuités ouvertes et antérieures au creusement de la carrière sont prises en compte. Les variations de ces paramètres sont donc décrites, du haut des versants (où se trouve l'entrée des carrières) vers l'intérieur des plateaux, sur des distances hectométriques (jusqu'à 500 m). Les résultats sont valorisés par des indices de fracturation calculés pour chaque carrière, de manière à connaître la densité du réseau de fissures des bordures des plateaux, et sa relation avec la morphologie de surface.

À l'échelle des hauts de versants, les carrières souterraines sont encore d'un grand secours. Dans les carrières de Ventelay, d'Hermonville 1 et de Branscourt 3, des relevés de bancs ont été effectués, à l'aide d'un niveau de chantier, sur une cinquantaine de mètres dans les galeries perpendiculaires au versant. Au sommet du Lutétien moyen, les niveaux de chailles brunes, de faluns et de calcaires durs sont suffisamment constants pour servir de repères (fig. 4). Ces relevés permettent de mesurer les décalages verticaux le long des fissures de détente, et les anomalies de pendage des blocs désolidarisés de la couche géologique, du fait de la proximité du versant.

\section{Résultats}

\section{La déformation à l'échelle des interfluves}

Les importantes variations, d'un interfluve à l'autre, de l'altitude et de la géométrie du mur des calcaires du Lutétien, ne s'expliquent pas uniquement par l'histoire tectonique du secteur. Dans un ensemble dont le pendage général ouest est de l'ordre de 0,2\% pour les assises tertiaires, M. Duprat (1997) décrit, à partir de la géométrie du toit de la craie campanienne et des variations d'épaisseur de la sédimentation tertiaire, une série de « cuvettes et hauts structuraux », associés à des déformations continues ou discontinues post-campaniennes. Cependant, ni le penda-

Fig. 5 - Géométrie du mur du Lutétien, du mur du Cuisien et du toit du Campanien. 1 : topographie ; 2 : mur du Lutétien ; 3 : mur du Cuisien ; 4 : toit du Campanien ; 5 : point de mesure ; 6 : sondage.

Fig. 5 - Geometry of the bottom of the Lutetian and the Cuisian, and of the top of the Campanian. 1: topography; 2: bottom of the Lutetian; 3: bottom of the Cuisian; 4: top of the Campanian; 5: measurement point; 6: borehole. ge général, ni les déformations secondaires ne rendent compte de la géométrie de détail du Lutétien. Plus encore, le Lutétien est le seul étage dont le mur ne soit pas parallèle au toit de la craie (fig. 5). Le dernier étage conforme est le Sparnacien, comme l'illustrent les coupes AA5, AC3, AC5

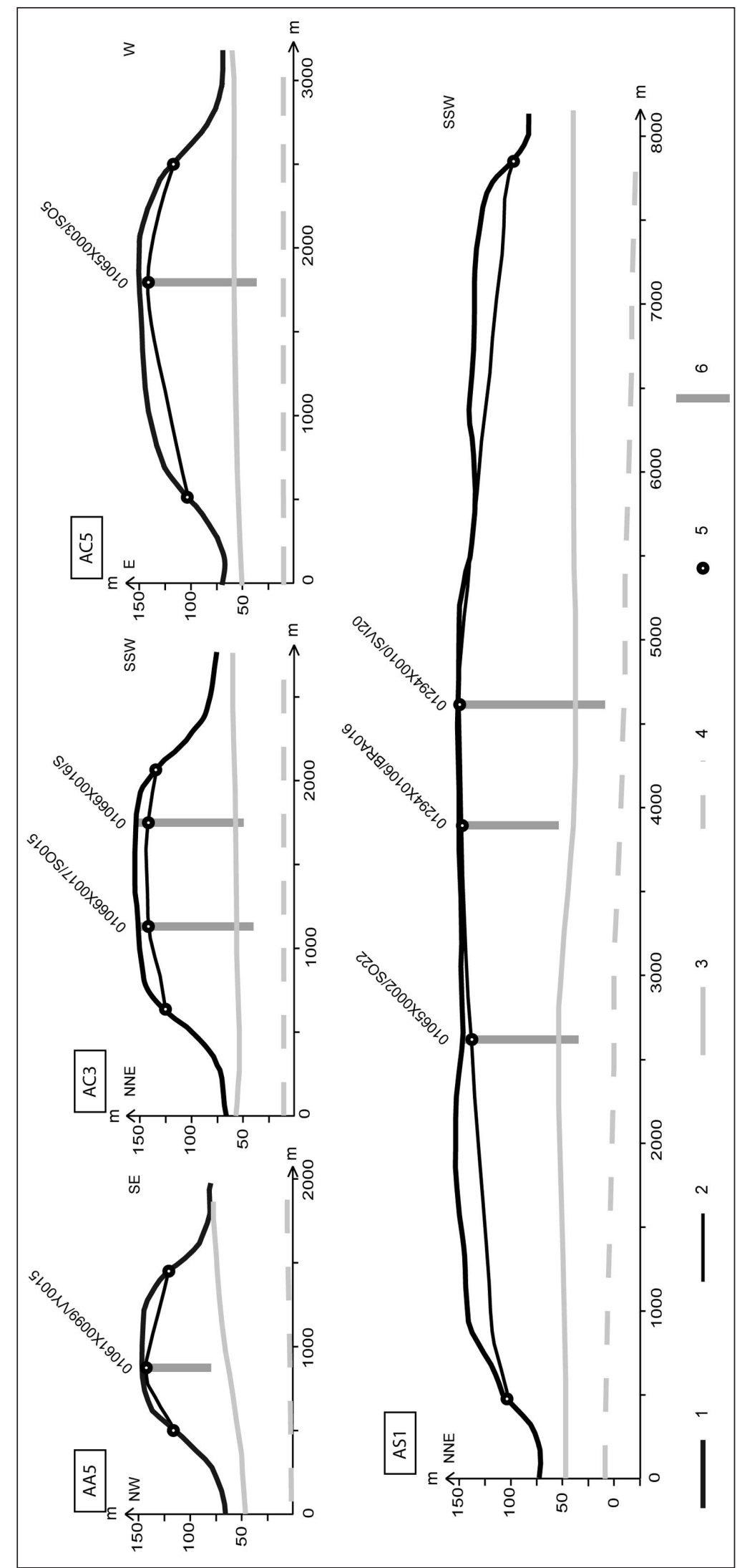


et $\mathrm{AS} 1$, où le toit des argiles sparnaciennes a le même pendage et les mêmes variations d'altitude que le toit de la craie campanienne, alors que le long des mêmes coupes, les calcaires lutétiens présentent un pendage non conforme (d'une valeur de 1,2\% vers le NNE et de 1,7 \% vers le SSW pour la coupe AS1, par exemple). De fait, à l'approche des vallées, le mur du Lutétien s'infléchit en moyenne de 3,3\%, avec un maximum de 9,3\% (coupe VA6) et un minimum de $1 \%$ (coupe CV2) (fig. 1 et fig. 5). On peut cependant observer une légère déformation anticlinale du toit du Sparnacien quand celui-ci affleure en fond de vallée (pendages divergents de l'ordre de 1 à $3 \%$ ), qui s'explique probablement par un phénomène de décompression lié au creusement de la vallée (Letourneur et Dayre, 1981). Au total, la déformation des couches est susceptible de se produire 1) à l'échelle de l'interfluve, où le gauchissement du Lutétien peut être imputable à un phénomène de décompression ; 2) à l'approche des versants, où les anomalies de pendage (ou l'exagération du pendage, sur les bordures de plateau tournées vers le centre du bassin de Paris) et l'abaissement de l'altitude du Lutétien traduisent un phénomène de détente, initiant une cambrure par appel au vide ; 3) dans le fond des vallées, où la décompression joue à nouveau. Dans les trois cas, ces modifications de la structure ne doivent rien aux déformations tectoniques régionales, d'origine interne.
2012). Cette densité de fissures cache en réalité une forte hétérogénéité spatiale due à la proximité du versant. La corrélation entre la distance au versant et l'indice de fracturation, qui détermine un nuage de points répondant à une loi exponentielle décroissante, illustre cette hétérogénéité (fig. 6). À l'entrée des bouches de cavage des carrières souterraines, c'est-à-dire au niveau du versant, le réseau de fissures est le plus dense avec des valeurs d'If supérieures à 0,3 fissure.m1, correspondant à des amplitudes inférieures à $5 \mathrm{~m}$. Pour une distance au versant comprise entre 0 et $150 \mathrm{~m}$, le degré de fracturation est important mais diminue fortement avant de se stabiliser, au-delà de $150 \mathrm{~m}$, autour d'une valeur d'If de 0,1 fissure. $\mathrm{m}^{-1}$, soit des amplitudes de plus de $15 \mathrm{~m}$. Cette variation spatiale de la fracturation témoigne non seulement d'une détente mécanique liée au creusement de la vallée mais aussi du contexte cassant dans lequel le fléchissement des calcaires s'inscrit.

Cependant, comme ces indices de fracturation sont calculés à partir de segments de droite perpendiculaires aux versants affectés par les fissures, ils intègrent toutes les discontinuités recoupées, dont celles proches du versant. Ils cachent donc des disparités spatiales au sein d'une même carrière. Pour illustrer l'organisation spatiale de la fracturation, les plans des carrières souterraines de Ventelay, d'Hermonville 1 et de Branscourt 3 ont été dressés (fig. 7). Les indices

\section{La déformation à l'échelle des rebords de plateau}

Les valeurs de fléchissement du Lutétien vers les vallées, mis en évidence par les altitudes du mur de la formation relevées en forage sur les plateaux et à l'affleurement dans les vallées, sont calculées à partir des données validées, mais trop souvent interprétées. Sur les versants, le substrat est généralement nappé d'épaisses formations superficielles masquant la structure. Dans ces conditions, la position du mur ou du toit des formations est parfois déduite à partir de critères morphologiques (Laurain et al., 1976), et reste donc imprécise. De plus, la répartition spatiale et la densité des forages contraignent à une interpolation hasardeuse des cotes NGF qui complique l'interprétation des données. La méthode d'interpolation choisie détermine un biais, interprété comme un fléchissement souple des couches géologiques vers les vallées. L'approche souterraine montre au contraire que ce fléchissement s'inscrit dans un réseau de fissures qui n'est dense qu'en bordure des plateaux. En effet, pour les 32 carrières topographiées, l'indice de fracturation au mètre linéaire (If), correspondant au nombre de fissures parallèles aux versants et recoupées par les galeries, est de 0,15 fissure. $\mathrm{m}^{-1}$, ce qui se traduit par une amplitude de $8,46 \mathrm{~m}$ entre chaque fissure (Devos et al.,

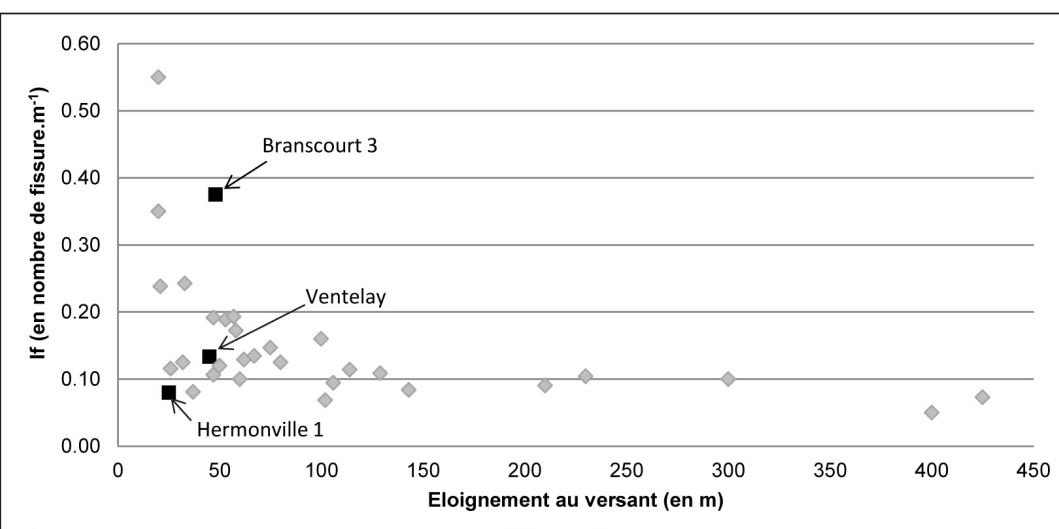

a.

-1 $\diamond 2$

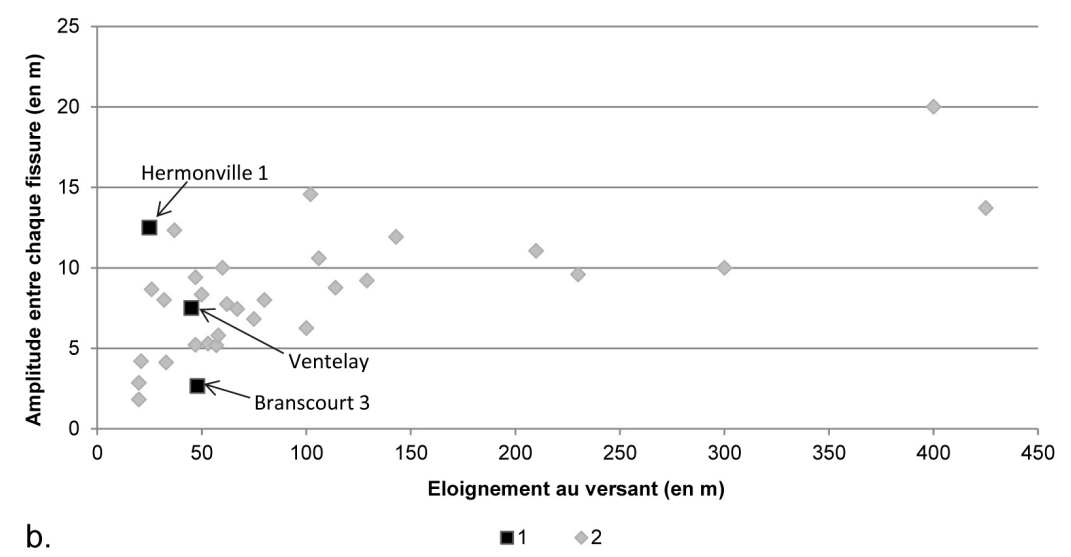

Fig. 6 - Indices de fracturation des carrières du Soissonnais et du Tardenois. 1 : carrière citée ; 2 : carrière étudiée.

Fig. 6 - Fracture index in quarries of Soissonnais and Tardenois. 1: quoted quarry; 2: studied quarry. 


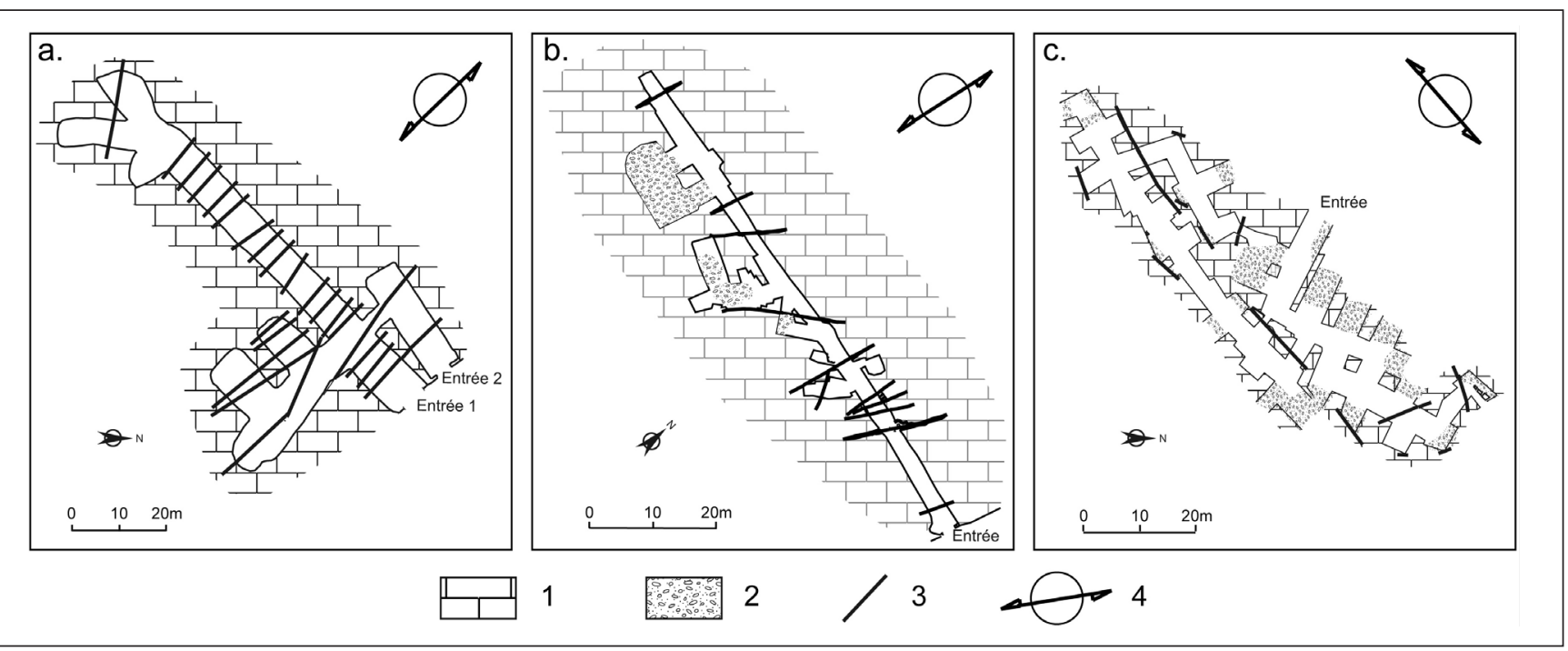

Fig. 7 - Topographie et réseaux de fissure. a : carrière de Branscourt $3 ; b$ : carrière de Ventelay ; $:$ carrière d'Hermonville $1 ; 1:$ calcaire (Lutétien) ; 2 : déblai ; 3 : fissure ; 4 : orientation de la vallée.

Fig. 7 - Topography and fissure network. a: quarry of Branscourt 3; b: quarry of Ventelay; c: quarry of Hermonville 1; 1: Lutetian limestone; 2: rubbish; 3: crack; 4: valley orientation.

de fracturation, dans les trois cas, s'intègrent bien dans la corrélation décrite plus haut, ce qui indique que les trois carrières sont représentatives de l'échantillon, et qu'elles s'inscrivent, en outre, sur la bordure des plateaux, à une cinquantaine de mètres du versant. À Ventelay et Branscourt (site de versant de vallée) la galerie principale, perpendiculaire au versant, recoupe 6 et 13 fissures sur une longueur de 45 et 48 m, respectivement. Pour ces deux carrières, le degré de fracturation est donc très important. À Hermonville (site de front de cuesta), l'organisation spatiale des fissures a guidé l'exploitation souterraine, comme c'est souvent le cas pour les carrières du XIX ${ }^{\mathrm{e}}$ siècle (Devos et al., 2010), ce qui détermine peu de recoupement de fissures par les galeries les plus longues, parallèles au versant (fig. 7c). L'azimut magnétique des fissures est clairement associé à celui du versant pour les trois carrières, ce qui permet de confirmer l'origine dynamique (c'est-à-dire liée à la détente), et non tectonique, de ces discontinuités. À l'image des carrières visitées, toutes les discontinuités correspondent à des fissures ouvertes dont la largeur (ou distance entre les blocs disjoints) est centimétrique (fig. 8), voire métrique. Certaines sont pénétrables mais la plupart est comblée par un remplissage de blocs hétérométriques dans une gangue argileuse provenant du soutirage des marnes et caillasses du Lutétien supérieur. L'ouverture des fissures résulte donc d'une détente mécanique et d'une extension latérale, conditionnées, en première approche, par un appel au vide.

Fig. 8 - Fissure et décalage de niveau repère à Hermonville. Le niveau de falun est décalé d'une vingtaine de centimètres, et l'ouverture de la fissure dans la partie basse indique un basculement des blocs.

Fig. 8 - Crack and displacement of key level. Displacement of twenty centimetres of a shelly limestone level. The open crack results of the tilting of the blocks.

\section{La déformation à l'échelle des hauts de versant}

À l'approche du haut de versant, où la densité de fissures est la plus élevée, des anomalies de pendage et des mouvements relatifs des blocs de calcaires lutétiens sont soulignés par les décalages de niveaux repères (chaille, falun et niveau

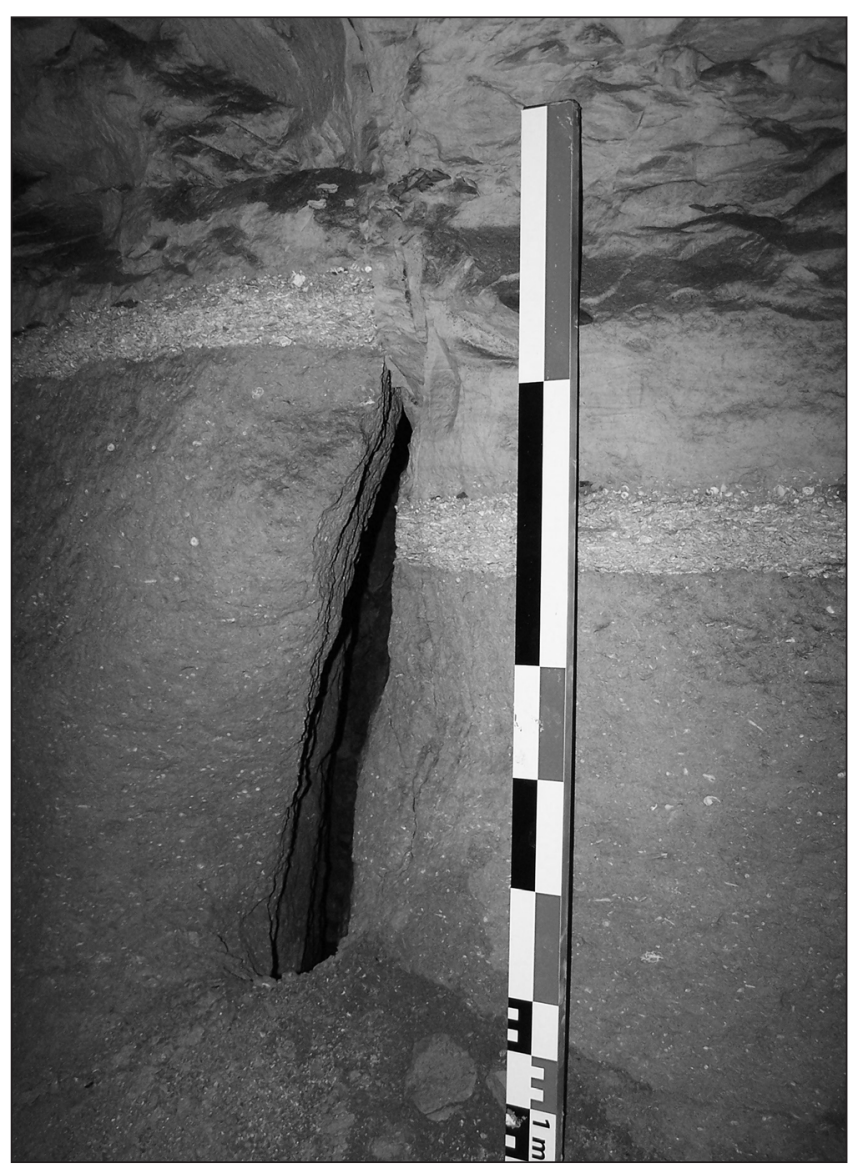


argileux) qui se suivent le long des galeries perpendiculaires au versant (fig. 4, fig. 8 et fig. 9). Pour les trois carrières souterraines retenues, ouvertes sur des versants regardant vers l'amont-pendage, les anomalies de pendage sont d'autant plus marquées que l'inclinaison des bancs, qui peut dépasser $15 \%$ (comme à Hermonville), n'est plus conforme au pendage régional (par ailleurs très faible : $0,2 \%$ ). Les niveaux repères mettent aussi en évidence des déplacements horizontaux et verticaux de part et d'autre des fissures séparant les blocs. Les pendages anormaux (dépassant $10 \%$ ) et les décalages verticaux (atteignant $60 \mathrm{~cm}$ ) sont les plus importants dans les carrières de Ventelay et Hermonville 1 , où le fléchissement des couches vers la vallée est le plus marqué. À Branscourt 3, les pendages $(\leq 4,5 \%)$ et les décalages verticaux (souvent inférieurs à $10 \mathrm{~cm}$ ) sont moins importants, et les jeux de blocs ne se font pas systématiquement en fonction de la pente du versant : aux basculements s'ajoutent des tassements. L'ouverture des fissures est variable (comprise entre $50 \mathrm{~cm}$ et $2 \mathrm{~m}$ ), et l'espace entre les blocs est rempli par des matériaux de soutirage. Enfin, l'examen détaillé des joints de stratification montre, par endroits, des décollements et des glissements banc sur banc d'ordre centimétrique. L'ensemble de ces déformations traduit un relâchement des contraintes qui affecte toutes les discontinuités des calcaires lutétiens, dont le volume augmente. Cette dilatance (Siriyes, 1984) se répercute dans les marnes et caillasses sus-jacentes qui subissent à leur tour des déformations, des tassements et du soutirage.

\section{Discussion}

\section{Tectonique, détente et décompression}

D'après les cartes géologiques, les plateaux du Tardenois et du Soissonnais ne sont pas faillés, cependant un ensemble de contraintes tectoniques post-campaniennes affectant l'Eocène a été déduit de l'étude des linéaments (Duprat, 1997). Il apparait que les vallées d'ordre 1 à 3 (classification de Horton) sont contraintes par les fracturations tectoniques d'orientation N 125

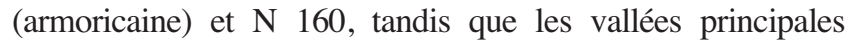
conservent un tracé est-ouest probablement hérité de la topographie fini-tertiaire de plaine, inclinée vers le centre du Bassin parisien (Dewolf et Pomerol, 1997). Dans ce dernier cas, la fracturation qui affecte les calcaires lutétiens est uniquement d'origine mécanique (« néofracturation »), tandis que le long des vallées secondaires, les fissures de détente se superposent à la trame tectonique. Au phénomène de détente, résultant d'un appel au vide sur les versants des vallées, il faut ajouter celui de décompression résultant d'une décharge et d'une minoration des contraintes lithostatiques (Letourneur et Dayre, 1981). La décompression, également susceptible de révéler des discontinuités virtuelles, affecte probablement le Lutétien en position d'interfluve, là où il est débarrassé des étages sus-jacents (Bartonien, Ludien et Stampien) ; elle est aussi à l'origine des déformations anticlinales décelées dans les argiles du Sparnacien en fond de vallée. Détente et décompression contribuent donc à l'apparition de structures superficielles dont il reste à évaluer le rôle dans la morphogenèse.

\section{Structure et modelé des versants}

En l'absence de mesures géophysiques et de sondages, l'interprétation de la structure des versants repose sur l'analyse de la topographie et des affleurements. Sur les communes de Jouy-lès-Reims, en front de cuesta, et de Janvry, sur le versant de rive gauche de la vallée de la Vesle (fig. 1), on observe des blocs de calcaires lutétiens reposant sur les sables cuisiens à des altitudes de $170 \mathrm{~m}$, soit près de $20 \mathrm{~m}$ plus bas qu'en position de plateau. Ces anomalies d'altitude ont déjà été constatées et interprétées en termes de tectonique (Laurent, 1908), ou de fauchage sur le haut de versant (Laurain et al., 1976). Ces deux hypothèses, cependant, ne rendent pas compte de la présence de blocs de Lutétien en fond de la vallée à $85 \mathrm{~m}$ d'altitude, soit $45 \mathrm{~m}$ plus bas que leur position initiale. C'est le cas, par endroits, dans la vallée de l'Ardre, où le Lutétien repose en outre sur les sables cuisiens, et non sur les argiles sparnaciennes qui affleurent dans le talweg, d'après la carte géologique (fig. 10). Sur les versants les plus longs de la rive droite de la vallée de l'Ardre, une série de ressauts, soulignés par des teintes blanchâtres sur les photographies aériennes, donne un aspect ondulé au versant (fig. 11) ; de l'avant-dernier ressaut, recoupé par la route, émergent des blocs de Lutétien, et les plus bas jouxtent la rivière.

Les orientations de pendage des blocs de calcaires lutétiens, dans leurs différentes positions topographiques, concordent avec celles relevées dans les Cotswold Hills et le Rutland où la cambrure de versant est très fréquente (Horswill et Horton, 1976 ; Chandler et al., 1976 ; Hobbs et Jenkins, 2008). Dans le Tardenois, les blocs qui jalonnent les versants au profil rectiligne et inclinés de $8^{\circ}$ à $10^{\circ}$, ont un pendage parallèle à la pente qu'ils drapent (drape profile), comme sur le front de cuesta et sur le versant de rive gauche de la Vesle. Le long des versants à replats et moins pentus $\left(5^{\circ}\right.$ à $\left.6^{\circ}\right)$, où s'observent parfois de légères contrepentes, les blocs sont au contraire relevant vers l'aval (back-tilted blocks). C'est le cas en rive droite de la vallée de l'Ardre, c'est-à-dire sur le versant dont la pente est conforme au pendage régional (fig. 10 et fig. 11). Enfin, les blocs de grandes dimensions qui atteignent les fonds de vallée au pied de versants courts, peuvent être plus redressés $\left(30^{\circ}\right)$, ce qui exprime un mouvement translationnel sur une pente décroissante, configuration également décrite dans les Cotswold Hills (Chandler et al., 1976). À cette structure de pied de versant est associé un replat, probablement perfectionné par un comblement colluvial. Il faut souligner que tous les versants de la vallée de l'Ardre ne présentent pas de cambrure continue : certains d'entre eux se terminent par une pente raide $\left(14^{\circ}\right)$ taillée dans les sables cuisiens. À ces endroits, où la vallée se resserre, le Lutétien ne forme cependant pas de corniche puisque, du fait de sa fracturation, il s'incline vers la vallée (hinge point des auteurs anglais).

\section{Morphogenèse}

Telle que décrite, la cambrure ne peut s'expliquer par un glissement de blocs calcaires sur les formations sous- 


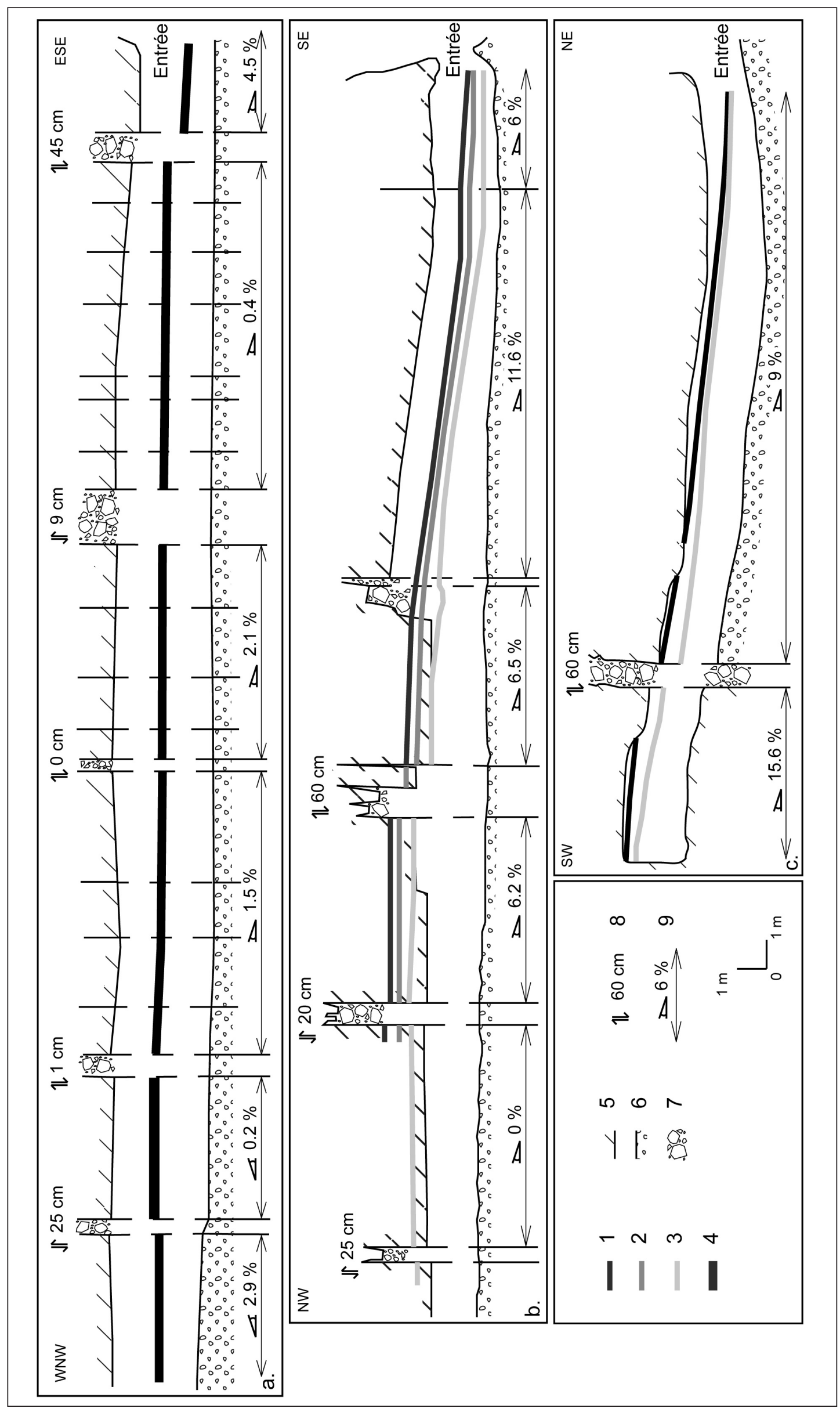

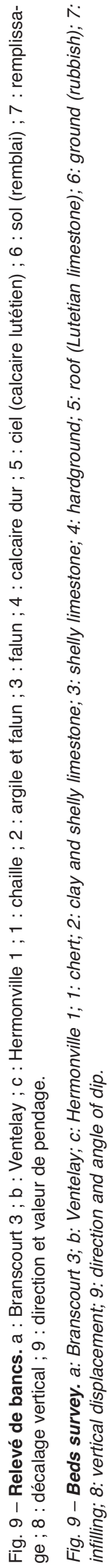




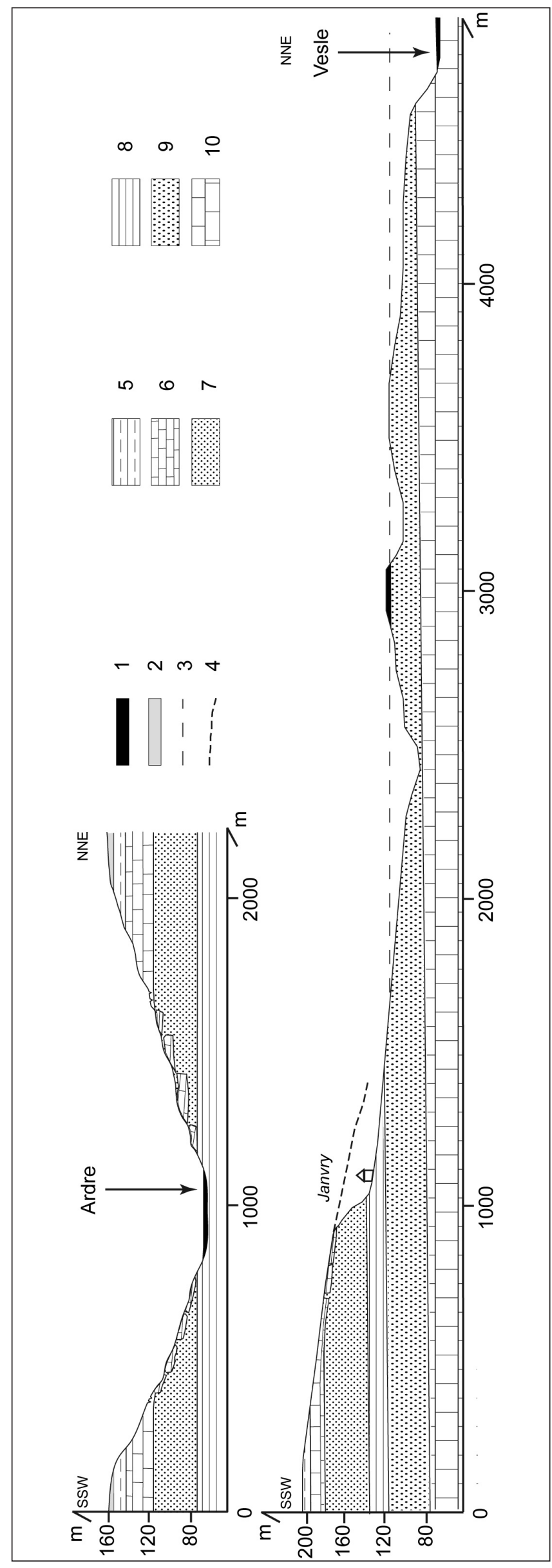

Fig. 10 - Coupes géomorphologiques interprétatives. 1 : formations colluvio-alluviales ; 2 : limons (Quaternaire) ; 3 : creusement saalien supposé ; 4 : cambrure tronquée ; 5 : Marnes et caillasses (Lutétien supérieur) ; $6:$ Calcaires grossiers (Lutétien inférieur et moyen) ; 7 : sable (Cuisien) ; 8 : argile (Sparnacien) ; 9 : sable (Thanétien) ; 10 : craie (Campanien).

Fig. 10 - Interpretative geomorphological sections. 1: colluvial and alluvial formations; 2: silt (Quaternary); 3: supposed Saalian cutting; 4: truncated cambering; 5: Marnes et caillasses (upper Lutetian); 6: Calcaires grossiers (lower and middle Lutetian); 7: sand (Cuisian); 8: clay (Sparnacian); 9: sand (Thanetian); 10: chalk (Campanian).

jacentes (Sosson et al., 2011), et ne se résume pas non plus à une extension latérale (Pasuto et Soldati, 1996), car ces deux processus ne rendent pas compte du fait que les blocs calcaires, pourtant désolidarisés, nappent en continuité les versants cambrés. Il faut donc envisager un abaissement progressif des strates, au fur et à mesure que la vallée se creuse, et cet abaissement ne peut s'expliquer que par le départ de matériaux sableux sous-jacents aux calcaires. Dans les structures où la roche incompétente est argileuse, cette crypto-érosion est attribuée au lessivage des roches meubles imperméables par les eaux de percolation (Hawkins et Privett, 1979), ou au fluage (Hollingworth et al., 1944), ou froissage (Hutchinson et Coope, 2002), conditionnés par un relâchement des contraintes sur les versants où les argiles affleurent, relâchement souligné par une bréchification (Vaughan, 1976 ; Hobbs et Jenkins, 2008). Dans le deuxième cas, il a également été suggéré que la déformation des matériaux est accélérée par la fonte d'un pergélisol (Parks, 1991 ; Hutchinson, 1991). Dans tous les cas, il en résulte un renflement du fond de vallée (valley bulging) où viennent s'accumuler les matériaux plastiques (fig. 3). Dans le Tardenois et le Soissonnais, le renflement des fonds de vallée est très peu marqué (le toit des argiles sparnaciennes est globalement parallèle au toit de la craie) et ne peut s'expliquer que par la décompression sensu stricto (c'est-à-dire sans appel au vide), d'autant que les versants sont essentiellement développés dans les sables cuisiens qui en contrôlent la dynamique (fig. 10). Comme, d'autre part, les matériaux sableux sont peu sensibles à la compaction (et donc à la décompaction), et sont par ailleurs perméables, leur déblaiement sous couverture calcaire ne peut se produire que dans des conditions de bilan hydrologique excédentaire favorable à l'infiltration (et au ruissellement), donc dans une ambiance périglaciaire. Ce dernier point fait consensus (Ballantyne et Harris, 1994), et la suffosion des sables cuisiens se produit en phase ana et/ou cataglaciaire.

Si la nécessité d'une ambiance froide implique un âge préholocène, la position perchée $(+100 \mathrm{~m})$ de certains versants cambrés (comme à Janvry, où la Vesle, en divaguant sur son fond plat, a fait reculer ses versants, fig. 10) plaide également en faveur d'une genèse ancienne, et pas nécessairement polycyclique (Horswill et Horton, 1976). Les quatre niveaux de terrasses alluviales de la Vesle (compris entre $\pm 5 \mathrm{~m}$ et $\pm 30 \mathrm{~m}$ ) ne s'apparentent pas nécessairement aux niveaux identifiés et datés le long de la haute Marne, et qui s'étagent sur une cinquantaine de mètres, car les deux secteurs n'ont pas la même 


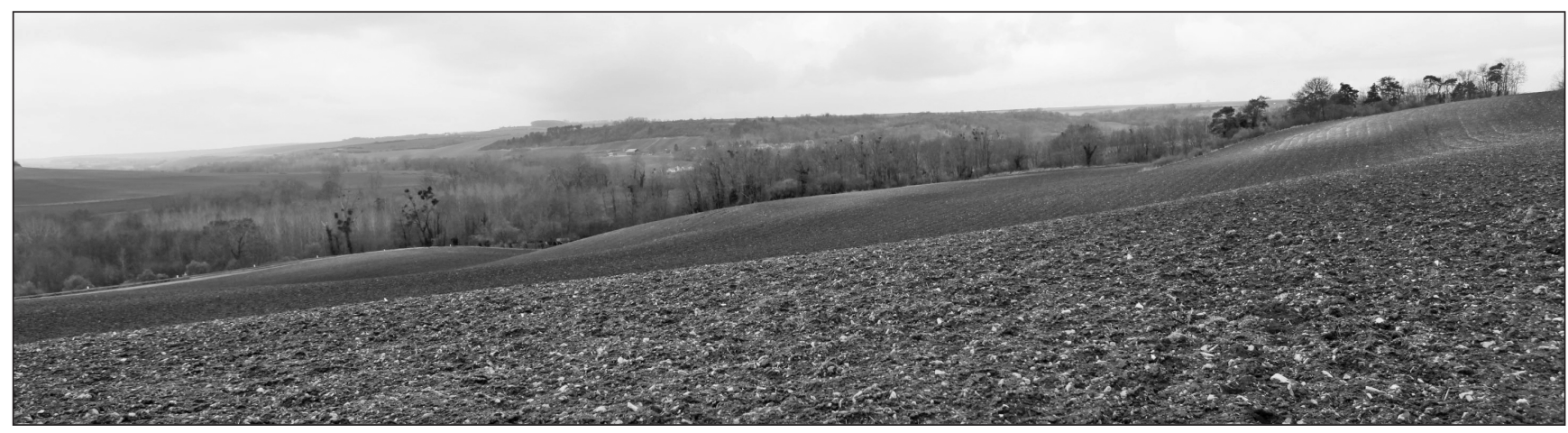

Fig. 11 - Vallée de l'Ardre. Au premier plan, versant à replats définis par la présence de blocs de calcaire lutétien relevant vers l'aval. Les taches blanchâtres correspondent au front des blocs sub-affleurants. Au deuxième plan, la surface du plateau du Tardenois.

Fig. 11 - Ardre valley. Foreground: ondulated valley side slope. The front of the back-tilted blocks of Lutetian limestone is underlined by a whitish shade. Background: Tardenois plateau.

histoire morphotectonique (Cojan et al., 2007). Les deux niveaux haut-marnais Ma3 $(+20-25 \mathrm{~m})$ et Ma2 $(+10-15 \mathrm{~m})$, datés de \pm 151000 ans et \pm 104000 ans (Lejeune, 2005), donnent cependant un ordre de grandeur qui concorde avec celui obtenu dans les Midlands (Horswill et Horton, 1976), permettant de conclure à une cambrure au moins anté-saalienne. Cette estimation s'accorde parfaitement avec les âges radiométriques obtenus sur des spéléothèmes postérieurs au phénomène de détente dans les calcaires bathoniens des Cotswold Hills, soit 350000 ans minimum (Self, 1995), impliquant un âge au moins elsterien pour la cambrure. Cet âge ancien signifierait que la cambrure de versant est une forme stable, se distinguant en cela des versants affectés par des glissements récurrents (Marre et al., 1997). R.J. Chandler et al. (1976) considèrent même la cambrure comme une forme fossile, épargnée par l'approfondissement subséquent de la vallée, ce qu'illustrerait la cambrure tronquée de Janvry (fig. 10). Le long de l'Ardre, dont l'étroit fond de vallée est typique d'un cours d'eau au profil longitudinal pentu, donc plus favorable à l'incision, la cambrure de versant reste intacte. Cette vallée illustre ainsi parfaitement les spécificités morphométriques (largeur de la grande base, longueur des versants et valeur des pentes) des vallées qui entaillent l'Eocène.

\section{Conclusion}

À l'est du bassin de Paris, seuls les versants des vallées des plateaux de l'Eocène sont affectés par une cambrure qui permet d'expliquer leur largeur importante. Ce processus est décelé à partir de critères morphologiques et de l'observation en carrière souterraine.

Contrairement aux Midlands anglais, les strates jurassiques du bassin de Paris se prêtent mal à la cambrure de versant, du fait notamment de la trop grande épaisseur des bancs calcaires. Ce n'est justement pas le cas dans l'Éocène de l'est de Paris, où le rapport d'épaisseur entre la couche compétente (Calcaires grossiers du Lutétien, 15 m à Fismes) et la couche incompétente (sables cuisiens, 20 à $30 \mathrm{~m}$ ) est très semblable à celui observé dans le Jurassique d'Angleterre (où l'oolite inférieure peu épaisse repose sur les Argiles du Lias ; Hobbs et Jenkins, 2008). Cette structure s'avère être plus propice à la cambrure de versant, qui se décline en : 1) détente mécanique, 2) extension latérale en haut de versant : initiation de la cambrure, et 3) cambrure : abaissement sur le versant de la strate dure de surface par érosion des couches meubles sous-jacentes. Cette suite de processus, classée dans la catégorie des « mouvements de versant complexes » (Selby, 1993), aboutit à des versants d'autant plus réguliers qu'ils sont recouverts par des formations gélifluidales de type head, et ils doivent à ce titre être distingués des versants dits lisses (Marre et al., 1997) consistant en glacis colluviaux, donc dus au ruissellement. Les versants cambrés du Soissonnais et du Tardenois méritent donc d'être élevés au rang de géomorphosite, d'autant que le phénomène de cambrure, replacé dans son contexte morphostructural (les plateaux éocènes), y est décrit en continuité du sommet de l'interfluve, grâce à l'apport de la subterranologie, au bas des versants, où une investigation géophysique et une prospection LiDAR permettraient de préciser la structure superficielle.

\section{Remerciements}

Les auteurs tiennent à remercier Peter Hobbs, du British Geological Survey, qui a relu la version anglaise abrégée, ainsi que J.-P. Peulvast et deux relecteurs anonymes pour leurs remarques.

\section{Références}

Ballantyne C.K., Harris C. (1994) - The periglaciation of Great Britain. Cambridge University Press, $330 \mathrm{p}$.

Blondeau A. (1965) - Le Lutétien des Bassin de Paris, de Belgique et du Hampshire. Thèse d'État, université de Paris, 467 p.

Buvignier A. (1852) - Statistique géologique, minéralogique, métallurgique et paléontologique du département de la Meuse. Baillière, Paris, 694 p.

Chandler R.J., Kellaway G.A., Skempton A.W. (1976) - Valley slope section in Jurassic strata near Bath, Somerset. Philosophical Transactions of the Royal Society of London, A, 283, 527-556.

Cojan I., Brulhet J., Corbonnois J., Devos A., Gargani J., Harmand D., Jaillet S., Jouve A., Laurain M., Lejeune O., Le Roux J., Losson B., Marre A., Moreau M.-G., Ricordel C., Simon-Coinçon R., Stab O., Thiry M., Voinchet P. (2007) - 
Morphologic evolution of eastern Paris Basin: «ancient surfaces» and Quaternary incisions. Mémoire de la Société Géologique de France, n.s., 178, 135-155.

Devos A., Chalumeau L., Bollot N., Sosson C., Barre E., Lejeune O., Gobancé G. (2012) - Organisation spatiale de la fracturation de détente mécanique de versant en carrières souterraines. Applications aux plateaux des calcaires éocènes du bassin de Paris. Physio-Géo - Géographie Physique et Environnement, 4, 243-262.

Devos A., Fronteau G., Lejeune O., Sosson C., Chopin E., Barbin V. (2010) - Influence of geomorphological constraints and exploitation techniques on stone quarry spatial organisation: Example of Lutetian underground quarries in Rheims, Laon and Soissons areas. Engineering Geology, 115, 268-275.

Dewolf Y., Pomerol C. (1997) - Surfaces d'érosion polygéniques dans les formations cénozoïques du Bassin parisien. Bulletin de la Société Géologique de France, 4, 473-482.

Dorigny A., Maucorps J., Pomerol C., Solau J.-L., Vatinel M. (1983) - Carte géologique de la France à 1/50 000. Feuille de Soissons, BRGM, Orléans, avec notice, $46 \mathrm{p}$.

Duprat M. (1997) - Modèle tectono-sédimentaire des dépôts paléogènes dans le nord-est du bassin de Paris : conséquences sur la géométrie du toit de la craie. Annales de la Société Géologique du Nord, 5, 269-287.

Fronteau G., Pascal A., Barbin V. (2002) - Caractérisation de la Pierre de Courville (Lutétien, Marne, France). Relations entre diagenèse et propriétés pétrophysiques. Géologie de la France, 4, 51-59.

Guilcher A., Tricart J. (1954) - La XXXVIe excursion géogra-

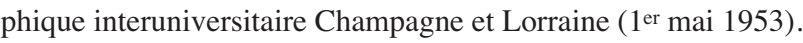
Annales de Géographie, 336, 88-98.

Hawkins A.B., Privett K.D. (1979) - Engineering geomorphological mapping as a technique to elucidate areas of superficial structures; with examples from the Bath area of the south Cotswolds. Quarterly Journal of Engineering Geology, 12, 221-234.

Hobbs P.R.N., Jenkins G.O. (2008) - Bath's 'foundered strata' a re-interpretation. British Geological Survey Research Report, OR/08/052. $40 \mathrm{p}$.

Hollingworth S.E., Taylor J.H., Kellaway G.A. (1944) - Largescale superficial structures in the Northampton Ironstone Field. Quarterly Journal of the Geological Society, 100, 1-44.

Horswill P., Horton A. (1976) - Cambering and valley bulging in the Gwash valley at Empingham, Rutland. Philosophical Transactions of the Royal Society of London, A, 283, 427-451.

Hutchinson J.N., Coope G.R. (2002) - Cambering and valley bulging, periglacial solifluction and Lateglacial Coleoptera at Dowdeswelle, near Cheltenham. Proceedings of the Geologists' Association, 113, 291-300.

Hutchinson J.N. (1991) - Periglacial and slope processes. Engineering Geology Special Publications, 7, Geological Society, London, 283-331.

Laurain M., Courtehoux H., Barta L., Guérin H. (1976) - Carte géologique de la France à 1/50 000. Feuille de Fismes, BRGM, Orléans, avec notice, $33 \mathrm{p}$.

Laurent J. (1908) - Le col de Pargny-Lès-Reims. Association Française pour l'Avancement des Sciences, compte rendu de la 36e session, Reims, 1907, 322-325.
Laurentiaux D., Barta L., Bergougnan H., Guérin H., Laurain M., Boureux M., Maucorps J. (1972) - Carte géologique de la France à 1/50 000. Feuille de Craonne, BRGM, Orléans, avec notice, $17 \mathrm{p}$.

Lejeune O. (2005) - Dynamique d'érosion des bas plateaux de l'Est de la France : l'exemple du basin-versant amont de la Marne. Thèse, université de Reims Champagne-Ardenne, t. 1, 472 p.

Letourneur J., Dayre M. (1981) - Aspects physico-mécaniques et incidences géotechniques de la décompression. Bulletin de l'Association de Géographes Français, 478, 133-138.

Marre A., Laurain M., Guérémy P. (1997) - Relations spatiales et temporelles entre les formations superficielles et les mouvements de terrain sur la côte de l'Île-de-France (Champagne) : un moyen de préparer les cartes des aléas. Géologie de la France, 2, 39-49.

Maubeuge P. (1949) - Un problème méconnu de géologie appliquée et de géographie physique : les structures superficielles. Bulletin de la Société des Sciences de Nancy, 2-3, 24-30.

Moiriat D., Potherat P., Durville J.-L., Bebien J. (2005) Observations sur la fracturation liée à l'incision d'une vallée carrière souterraine des brillants (Hauts-de-Seine), Bulletin des Laboratoires des Ponts et Chaussées, 258-259, 3-14.

Parks C.D. (1991) - A review of the mechanisms of cambering and valley bulging. Geological Society, London, Engineering Geology Special Publications, 7, 373-380.

Pasuto A., Soldati M. (1996) - Rock spreading. In Dikau R., Brunsden D., Schrott L., Ibsen M.-L. (Eds.) Landslide recognition. Wiley, 122-136.

Salin R., Dorigny A., Vatinel M., Pomerol C. (1977) - Carte géologique de la France à 1/50 000. Feuille de Fère-en-Tardenois, BRGM, Orléans, avec notice, 18 p.

Selby M.J. (1993) - Hillslope materials and processes. Oxford University Press, $451 \mathrm{p}$.

Self C.A. (1995) - The relationship between the gull cave Sally's rift and the development of the river Avon east of Bath. Proceedings of the University of Bristol Spelaeological Society, 20, 91-108.

Sirieys P. (1984) - La dilatance des roches. Revue Française de Géotechnique, 27, 23-25.

Sosson C., Devos A., Lejeune O., Marre A. (2011) - Apport des observations en carrière souterraine dans la compréhension des dynamiques géomorphologiques des plateaux tertiaires du Bassin de Paris Exemple du Tardenois, du Soissonnais et du Valois (Aisne, France). In M.-J. Penven, H. Régnauld, D. Mercier (Eds.), Mobilité des formes et surfaces terrestres. Presses Universitaires de Rennes, 145-164.

Tricart J. (1952) - La partie orientale du bassin de Paris. t. 2 : L'évolution morphologique au Quaternaire. SEDES, 474 p.

Tricart J. (1977) - Précis de géomorphologie. t. 2 : Géomorphologie dynamique générale. SEDES CDU, 345 p.

Van Den Eeckaut M., Marre A., Poesen J. (2010) - Comparison of two landslide susceptibility assessments in the ChampagneArdennes region (France). Geomorphology, 115, 141-155.

Vaughan P.R. (1976) - The deformation of the Empingham valley slope. Philosophical Transactions of the Royal Society of London, A, 283, 451-462.

Article soumis le 10 avril 2013, accepté le 8 octobre 2013. 